\title{
A Search for Vector Diquarks at the CERN LHC
}

\author{
E. Arik ${ }^{a}$, O. Çakır ${ }^{b}$, S. A. Çetin ${ }^{a}$ and S. Sultansoy ${ }^{c, d}$ \\ ${ }^{a}$ Bogazici University, Faculty of Sciences, Department of Physics, \\ 80815, Bebek, Istanbul, Turkey \\ ${ }^{b}$ Ankara University, Faculty of Sciences, Department of Physics, \\ 06100 Tandogan, Ankara, Turkey \\ ${ }^{c}$ Gazi University, Faculty of Arts and Sciences, Department of Physics, \\ 06500, Teknikokullar, Ankara, Turkey \\ $d$ Azerbaijan Academy of Sciences, Institute of Physics, \\ H. Cavid av., 33, Baku, Azerbaijan
}

\begin{abstract}
Resonant production of the first generation vector diquarks at the CERN Large Hadron Collider (LHC) is investigated. It is shown that the LHC will be able to discover vector diquarks with masses up to $9 \mathrm{TeV}$ for quark-diquark-quark coupling $\alpha_{D}=0.1$ and $4 \mathrm{TeV}$ for $\alpha_{D}=5 \times 10^{-4}$.
\end{abstract}

The existence of at least three fermion families and especially inter-family mixings naturally lead to the hypothesis that they are made up of more fundamental constituents frequently called preons [1]. Today, the compositeness should be considered as a candidate for beyond the standard model (BSM) physics on the same footing as SUSY. Moreover, some assumptions made in order to get rid of huge number of free parameters in three family minimal supersymmetric standard model (MSSM) have natural explanation (see [2] and references therein) in the framework of preonic models. These models predict a rich spectrum of new particles with unusual quantum numbers at high energies such as excited quarks and leptons, diquarks, dileptons, leptoquarks, leptogluons, sextet quarks, octet bosons etc. In preonic models, diquarks are as natural as leptoquarks [3]. They are colored objects having integer-spin and baryon number $|B|=2 / 3$ or 0 . Diquarks are also predicted in the framework of superstring-inspired $E_{6}$ models [4].

Recent collider limits on the diquark masses come from Tevatron data which exclude the region $290<M_{D}<420 \mathrm{GeV}$ (for $\mathrm{E}_{6}$ diquarks) [5]. Diquark production at $e^{+} e^{-}$colliders, $e p$ colliders and $p \bar{p}$ colliders have been analyzed in [6], [7] 
and [8] respectively. The resonance production of scalar diquarks at CERN LHC has been studied in [9].

In this work, we study composite vector diquark production at the LHC. Interaction Lagrangian and quantum numbers of diquarks are discussed. Decay width and production cross section of vector diquarks at the LHC are considered. Vector diquark signal and corresponding backgrounds are analyzed.

A model independent, baryon number conserving, most general $S U(3)_{C} \times$ $S U(2)_{W} \times U(1)_{Y}$ invariant effective Lagrangian for diquarks has the form

$$
\begin{aligned}
L_{|B|=0}= & f_{1 L} \bar{q}_{L} \gamma^{\mu} q_{L} D_{1 \mu}^{c}+\left(f_{1 R} \bar{d}_{R} \gamma^{\mu} d_{R}+f_{1 R}^{\prime} \bar{u}_{R} \gamma^{\mu} u_{R}\right) D_{1 \mu}^{\prime c} \\
& +\widetilde{f}_{1 R} \bar{u}_{R} \gamma^{\mu} d_{R} \widetilde{D}_{1 \mu}^{c}+f_{3 L} \bar{q}_{L} \tau \gamma^{\mu} q_{L} \cdot \mathbf{D}_{3 \mu}^{c} \\
& +f_{2} \bar{q}_{L} i \tau_{2} u_{R} D_{2}^{c}+\widetilde{f}_{2} \bar{q}_{L} i \tau_{2} d_{R} \widetilde{D}_{2}^{c}+H . c . \\
L_{|B|=2 / 3}= & \left(g_{1 L} \bar{q}_{L}^{c} i \tau_{2} q_{L}+g_{1 R} \bar{u}_{R}^{c} d_{R}\right) D_{1}^{c}+\widetilde{g}_{1 R} \bar{d}_{R}^{c} d_{R} \widetilde{D}_{1}^{c} \\
& +\widetilde{g}_{1 R}^{\prime} \bar{u}_{R}^{c} u_{R} \widetilde{D}_{1}^{\prime c}+g_{3 L} \bar{q}_{L}^{c} i \tau_{2} \tau q_{L} \cdot \mathbf{D}_{3}^{c} \\
& +g_{2} \bar{q}_{L}^{c} \gamma^{\mu} d_{R} D_{2 \mu}^{c}+\widetilde{g}_{2} \bar{q}_{L}^{c} \gamma^{\mu} u_{R} \widetilde{D}_{2 \mu}^{c}+H . c .
\end{aligned}
$$

Diquarks with baryon number $B=0$ are familiar fields, as they resemble the electroweak gauge vectors, and the neutral and charged Higgs scalars. Here, we consider only $|B|=2 / 3$ vector diquarks. In Eq. (2), $q_{L}=\left(u_{L}, d_{L}\right)$ and $q^{c}=C \bar{q}^{T}\left(\bar{q}^{c}=-q^{T} C^{-1}\right)$. For the sake of simplicity, color and generation indices are omitted. Scalar diquarks $D_{1}, \tilde{D}_{1}, \tilde{D}_{1}^{\prime}$ are $S U(2)_{W}$ singlets and $\mathbf{D}_{3}$ is $S U(2)_{W}$ triplet. Vector diquarks $D_{2}$ and $\tilde{D}_{2}$ are $S U(2)_{W}$ doublets. Diquarks may transform as anti-triplet or sextet under $S U(3)_{C}$. At this stage, we assume that each SM generation has its own diquarks and couplings in order to avoid flavour changing neutral currents.

Lagrangian (2) can be rewritten in the following more transparent form:

$$
\begin{aligned}
L & =L_{S}+L_{V} \\
L_{S} & =\left[g_{1 L}\left(\bar{u}^{c} P_{L} d-\bar{d}^{c} P_{L} u\right)+g_{1 R} \bar{u}^{c} P_{R} d\right] D_{1}+\tilde{g}_{1 R} \bar{d}^{c} P_{R} d \tilde{D}_{1} \\
& +\tilde{g}_{1 R}^{\prime} \bar{u}^{c} P_{R} u \tilde{D}_{1}^{\prime}+\sqrt{2} g_{3 L} \bar{u}^{c} P_{L} u D_{3}^{+}-\sqrt{2} g_{3 L} \bar{d}^{c} P_{L} d D_{3}^{-} \\
& -g_{3 L}\left(\bar{u}^{c} P_{L} d+\bar{d}^{c} P_{L} u\right) D_{3}^{0}+H . c . \\
L_{V} & =g_{2} \bar{u}^{c} \gamma^{\mu} P_{R} d D_{2 \mu}^{1 c}+g_{2} \bar{d}^{c} \gamma^{\mu} P_{R} d D_{2 \mu}^{2 c}+\tilde{g}_{2} \bar{u}^{c} \gamma^{\mu} P_{R} u \tilde{D}_{2 \mu}^{1 c} \\
& +\tilde{g}_{2} \bar{d}^{c} \gamma^{\mu} P_{R} u \tilde{D}_{2 \mu}^{2 c}+H . c .
\end{aligned}
$$

where $P_{L}=\left(1-\gamma_{5}\right) / 2$ and $P_{R}=\left(1+\gamma_{5}\right) / 2$. A general classification of the first generation, color $\overline{3}$ diquarks is shown in Table 1 .

For the present analysis, we consider the color $\overline{3}$ vector diquark $D_{2}$ with charge $1 / 3$ which couples to $u d$-pair as described by the effective Lagrangian (5). The decay width $\Gamma_{D}$, derived from the same Lagrangian, is 


$$
\Gamma_{D}=\frac{\alpha_{D} M_{D}}{9} \simeq 11 \mathrm{GeV}\left(\frac{M_{D}}{1 T e V}\right) \text { for } \alpha_{D}=0.1,
$$

where $\alpha_{D}=g_{2}^{2} / 4 \pi, M_{D}$ is the mass of vector diquark. We use the narrow width approximation and consider this as long as $\Gamma_{D} / M_{D}<0.1$. The cross section of the s-channel diquark resonance production can be obtained as

$$
\sigma\left(p p \rightarrow D_{2} X\right)=\sigma_{0} \int_{M_{D}^{2} / s}^{1} \frac{d x}{x} f_{u}\left(x, Q^{2}\right) f_{d}\left(\frac{M_{D}^{2}}{s x}, Q^{2}\right)
$$

with

$$
\sigma_{0}=\frac{8 \pi^{2} \alpha_{D}}{9 s}
$$

where the factorization scale $Q^{2}=M_{D}^{2}, f_{u}$ and $f_{d}$ are quark distribution functions of each proton. In Fig. 1, using the CTEQ5L quark distribution functions [10], the cross section versus diquark mass is plotted for LHC energy $(\sqrt{s}=14 \mathrm{TeV})$ with $\alpha_{D}=0.1$.

$D_{2}$-type diquark will decay via $D_{2} \rightarrow u d$. Therefore, the signal will contain two hard jets in the final state. At LHC energy, major QCD processes contributing to two jet $(j j)$ final states and their integrated cross sections are given in Table 2. The values in Table 2 have been generated by PYTHIA 6.1 [11] at parton level with various $p_{T}$ cuts. It is clear that higher $p_{T}$ cuts reduce the background cross sections significantly. These $p_{T}$ cuts can be translated into the rapidity cuts via the relation between the $p_{T}$ of a jet and the rapidity $y$ given by $p_{T}=M_{j j} / 2 \cosh y$.

The differential cross section as a function of the dijet invariant mass $M_{j j}$, with the rapidity cut $\left|y_{1,2}\right| \leq Y$, where $y_{1}$ and $y_{2}$ are rapidities of the massless final quarks, is given by

$$
\begin{aligned}
\frac{d \sigma}{d M_{j j}} & =\frac{M_{j j}^{3}}{2 s} \int_{-Y}^{Y} d y_{2} \int_{y_{1}^{\min }}^{y_{1}^{\max }} d y_{1} \frac{1}{\cosh ^{2} y^{\star}} \\
& \times\left[f_{u / A}\left(x_{u}, Q^{2}\right) f_{d / B}\left(x_{d}, Q^{2}\right) \frac{d \hat{\sigma}}{d \hat{t}}(\hat{s}, \hat{t}, \hat{u})\right. \\
& \left.+f_{d / A}\left(x_{d}, Q^{2}\right) f_{u / B}\left(x_{u}, Q^{2}\right) \frac{d \hat{\sigma}}{d \hat{t}}(\hat{s}, \hat{u}, \hat{t})\right]
\end{aligned}
$$

with

$$
\begin{aligned}
x_{u} & =\sqrt{\tau} e^{y^{b}}, \quad x_{d}=\sqrt{\tau} e^{-y^{b}} \\
\hat{s} & =x_{u} x_{d} s, \quad \hat{t}=-x_{u} p_{T} \sqrt{s} e^{-y_{1}}, \quad \hat{u}=-x_{d} p_{T} \sqrt{s} e^{y_{1}} \\
y^{\star} & =\left(y_{1}-y_{2}\right) / 2, \quad y^{b}=\left(y_{1}+y_{2}\right) / 2 \\
y_{1}^{\min } & =\max \left(-Y, \log \tau-y_{2}\right), \quad y_{1}^{\max }=\min \left(Y,-\log \tau-y_{2}\right), \quad \tau=M_{j j}^{2} / s .
\end{aligned}
$$


In Eq. (9), the differential cross section for the subprocess $u d \rightarrow u d$ has the form

$$
\begin{aligned}
\frac{d \hat{\sigma}}{d \hat{t}}(u d \rightarrow u d)= & \frac{4 \pi}{9}\left[\frac{\alpha_{D}^{2}(\hat{s}+\hat{t})^{2}}{\hat{s}^{2}\left[\left(\hat{s}-M_{D}^{2}\right)^{2}+M_{D}^{2} \Gamma_{D}^{2}\right]}\right. \\
& \left.+\frac{2 \alpha_{s}^{2}}{\hat{s}^{2}} \frac{\left(2 \hat{s}^{2}+\hat{t}^{2}+2 \hat{s} \hat{t}\right)}{\hat{t}^{2}}-\frac{2 \alpha_{D} \alpha_{s}}{\hat{t}} \frac{(\hat{s}+\hat{t})^{2}\left(\hat{s}-M_{D}^{2}\right)}{\hat{s}^{2}\left[\left(\hat{s}-M_{D}^{2}\right)^{2}+M_{D}^{2} \Gamma_{D}^{2}\right]}\right]
\end{aligned}
$$

where the dominant interference with the the t-channel gluon exchange is taken into account.

Fig. 2 shows jet-jet invariant mass distribution for the process $p p \rightarrow D_{2} \rightarrow$ $j j X$ together with the estimations of the QCD backgrounds at LHC. For comparison, signal peaks for vector diquark masses $M_{D}=2,4,6,8 \mathrm{TeV}$ and $\alpha_{D}=0.1$ are superimposed on the background distribution.

We have estimated background events for an integrated LHC luminosity of $10^{5} \mathrm{pb}^{-1}$ taking into account, as an example, the energy resolution of ATLAS hadronic calorimeter [12]:

$$
\frac{\delta E}{E}=\frac{50 \%}{\sqrt{E}}+3 \%
$$

for jets with $|y|<3$. The mass resolution can be expressed as

$$
\frac{\Delta M}{M} \approx \frac{\delta E}{\sqrt{2} E}
$$

We have chosen $\Delta M$ as the mass window centered at $M_{D}$ for signal and background estimations. Here, we take the energy of a jet $E \approx M_{D} / 2$. For signal estimation the mass window $\Delta M$ is taken to be $2 \Gamma_{D}$. This corresponds to the 95\% CL for statistical acceptance. The cross sections are calculated by using the formula

$$
\sigma=\int_{M_{D}-\Delta M / 2}^{M_{D}+\Delta M / 2} d M_{j j}\left(\frac{d \sigma}{d M_{j j}}\right)
$$

Number of signal $(S)$ and background $(B)$ events, and the corresponding significances with $\alpha_{D}=0.1$ are tabulated in Table 3. Evidently, tighter cut on the rapidity $y$ improves the significance considerably. In Table 4 , we present the achievable $M_{D}$ limits for different values of $\alpha_{D}$. As discovery criteria, we adopt $S / \sqrt{B} \geq 5$ and $S \geq 25$.

In Fig. 3, the attainable mass limits are presented in the plane $\left(\alpha_{D}, M_{D}\right)$. The LHC potential for the discovery of $D_{2}$ is clearly demonstrated in Fig. 田 where minimum integrated luminosities, needed to satisfy the adopted criteria, are plotted as a function of $M_{D}$ for various $\alpha_{D}$ values. 
There are ten different diquarks listed in Table 1, coupled to the first family quarks. All of them will decay into 2 jet final states. Vector and scalar type diquarks can be easily distinguished by the angular distribution of produced jets. To identify different vector (or scalar) diquarks at hadron colliders, one needs polarized proton beams. In principle, electric charge of diquarks can be determined at future lepton and photon colliders, provided that the center of mass energy is larger than $2 M_{D}$. Furthermore, $\gamma p$ colliders based on linac-ring type $e p$ colliders will give essential contribution to the subject. The observation of the associated production of diquarks with leptoquarks will be possible at future lepton-hadron colliders.

In conclusion, the resonance production of vector diquarks at LHC has large cross section. With reasonable cuts, it may be possible to cover mass ranges up to $9 \mathrm{TeV}$ for coupling $\alpha_{D}=0.1$. For smaller couplings as $\alpha_{D}=5 \times 10^{-4}$, it is still possible to probe diquarks up to the mass of $4 \mathrm{TeV}$ at an integrated luminosity $L=10^{2} \mathrm{fb}^{-1}$.

\section{References}

[1] H. Terazawa, Phys. Rev. 22 (1980) 184; H. Harari, Phys. Rep. 104 (1984) 159; D'Souza and Kalman, Preons, World Scientific Publishing, (1992).

[2] S. Sultansoy, hep-ph/0003269 (2000).

[3] J. Wudka, Phys. Lett. B167 (1986) 337.

[4] J.L. Hewett and T.G. Rizzo, Phys. Rep. 183 (1989) 193.

[5] F. Abe et al., CDF Collaboration, Phys. Rev. Lett. 77 (1996) 5336; B. Abbott et al., D0 Collaboration, Phys. Rev.. Lett. 80 (1998) 666.

[6] D. Schaile and P.M. Zerwas: Proceedings of the Workshop on Physics at Future Accelerators, CERN Yellow Report 87-07, Vol. II, p.251 (1987).

[7] T.G. Rizzo, Z. Phys. C43 (1989) 223.

[8] V.D. Angelopoulos et al., Nucl. Phys. B292, 59 (1987).

[9] S. Atağ, O. Çakır, and S. Sultansoy, Phys. Rev. D59, 015008 (1998).

[10] CTEQ Collaboration, H.L. Lai et al., Eur.Phys. J. C12 (2000) 375.

[11] Torbjörn Sjöstrand et.al hep-ph/0010017 (2000).

[12] ATLAS Collaboration, ATLAS TDR 14, CERN/LHCC 99-14 (1999). 
Table 1: Quantum numbers of the first generation, color $\overline{3}$ and baryon number $|B|=2 / 3$ diquarks described by the effective Lagrangian (5) according to $S U(2)_{W} \times U(1)_{Y}$ invariance. $Q_{e m}=I_{3}+Y / 2$

\begin{tabular}{ccccc}
\hline Scalar Diquarks & $\mathrm{SU}(2)_{W}$ & $\mathrm{U}(1)_{Y}$ & $Q_{\text {em }}$ & Couplings \\
\hline$D_{1}$ & 1 & $2 / 3$ & $1 / 3$ & $u_{L} d_{L}\left(g_{1 L}\right), u_{R} d_{R}\left(g_{1 R}\right)$ \\
\hline$\tilde{D}_{1}$ & 1 & $-4 / 3$ & $-2 / 3$ & $d_{R} d_{R}\left(\tilde{g}_{1 R}\right)$ \\
\hline$\tilde{D}_{1}^{\prime}$ & 1 & $8 / 3$ & $4 / 3$ & $u_{R} u_{R}\left(\tilde{g}_{1 R}^{\prime}\right)$ \\
\hline$D_{3}$ & 3 & $2 / 3$ & $\left(\begin{array}{c}4 / 3 \\
1 / 3 \\
-2 / 3\end{array}\right)$ & $\left(\begin{array}{c}u_{L} u_{L}\left(\sqrt{2} g_{3 L}\right) \\
u_{L} d_{L}\left(-g_{3 L}\right) \\
d_{L} d_{L}\left(-\sqrt{2} g_{3 L}\right)\end{array}\right)$ \\
\hline Vector Diquarks & \multicolumn{1}{|c}{} \\
\hline$D_{2}$ & 2 & $-1 / 3$ & $\left(\begin{array}{c}1 / 3 \\
-2 / 3\end{array}\right)$ & $\left(\begin{array}{c}d_{R} u_{L}\left(g_{2}\right) \\
d_{R} d_{L}\left(-g_{2}\right)\end{array}\right)$ \\
\hline$\tilde{D}_{2}$ & 2 & $5 / 3$ & $\left(\begin{array}{c}4 / 3 \\
1 / 3\end{array}\right)$ & $\left(\begin{array}{c}u_{R} u_{L}\left(\tilde{g}_{2}\right) \\
u_{R} d_{L}\left(-\tilde{g}_{2}\right)\end{array}\right)$ \\
\hline
\end{tabular}

Table 2: Cross sections (in pb) for QCD backgrounds contributing to 2 jets final states at parton level, generated by PYTHIA 6.1 with various $p_{T}$ cuts.

\begin{tabular}{lcccc}
\hline Process & $p_{T}>100 \mathrm{GeV}$ & $p_{T}>500 \mathrm{GeV}$ & $p_{T}>1000 \mathrm{GeV}$ & $p_{T}>2000 \mathrm{GeV}$ \\
\hline$g g \rightarrow g g$ & $6.3 \times 10^{5}$ & $2.0 \times 10^{2}$ & $2.3 \times 10^{0}$ & $5.7 \times 10^{-3}$ \\
$q_{i} g \rightarrow q_{i} g$ & $6.4 \times 10^{5}$ & $4.8 \times 10^{2}$ & $1.0 \times 10^{1}$ & $5.7 \times 10^{-2}$ \\
$q_{i} q_{j} \rightarrow q_{i} q_{j}$ & $1.0 \times 10^{5}$ & $1.8 \times 10^{2}$ & $6.7 \times 10^{0}$ & $8.8 \times 10^{-2}$ \\
$g g \rightarrow q_{k} \bar{q}_{k}$ & $2.4 \times 10^{4}$ & $9.8 \times 10^{0}$ & $1.0 \times 10^{-1}$ & $2.9 \times 10^{-4}$ \\
$q_{i} \bar{q}_{i} \rightarrow q_{k} \bar{q}_{k}$ & $1.6 \times 10^{3}$ & $2.8 \times 10^{0}$ & $1.3 \times 10^{-1}$ & $1.1 \times 10^{-3}$ \\
$q_{i} \bar{q}_{i} \rightarrow g g$ & $1.5 \times 10^{3}$ & $2.5 \times 10^{0}$ & $6.7 \times 10^{-2}$ & $8.5 \times 10^{-4}$ \\
\hline Total & $1.4 \times 10^{6}$ & $8.8 \times 10^{2}$ & $1.9 \times 10^{1}$ & $1.5 \times 10^{-1}$ \\
\hline
\end{tabular}


Table 3: Observability of the vector diquark $D_{2}$ with $\alpha_{D}=0.1$ at LHC for $L_{\text {int }}=10^{5} \mathrm{pb}^{-1}$.

\begin{tabular}{lccccc}
\hline$M_{D}(\mathrm{TeV})$ & 1 & 3 & 5 & 7 & 9 \\
\hline$\left|y_{1,2}\right|<2$ & & & & & \\
\hline$S$ & $7.7 \times 10^{7}$ & $1.1 \times 10^{6}$ & $5.0 \times 10^{4}$ & $1.7 \times 10^{3}$ & $2.7 \times 10^{1}$ \\
$B$ & $2.8 \times 10^{8}$ & $4.2 \times 10^{5}$ & $8.4 \times 10^{3}$ & $2.2 \times 10^{2}$ & $3.1 \times 10^{0}$ \\
$S / \sqrt{B}$ & 4585 & 1768 & 545 & 118 & 16 \\
\hline$\left|y_{1,2}\right|<1$ & & & & & \\
\hline$S$ & $2.6 \times 10^{7}$ & $5.8 \times 10^{5}$ & $2.9 \times 10^{4}$ & $1.1 \times 10^{3}$ & $1.8 \times 10^{1}$ \\
$B$ & $2.5 \times 10^{7}$ & $4.0 \times 10^{4}$ & $7.9 \times 10^{2}$ & $2.1 \times 10^{1}$ & $3.0 \times 10^{-1}$ \\
$S / \sqrt{B}$ & 5213 & 2889 & 1044 & 245 & 34 \\
\hline
\end{tabular}

Table 4: Achievable diquark mass limits for different quark-diquark-quark couplings in the framework of discovery criteria given in the text. $\left|y_{1,2}\right|<2$.

\begin{tabular}{ccccc}
\hline$\alpha_{D}$ & $M_{D}(\mathrm{TeV})$ & $\mathrm{S}$ & $\mathrm{B}$ & $\mathrm{S} / \sqrt{B}$ \\
\hline 0.1 & 9 & 27 & 3.1 & 16.0 \\
0.01 & 8 & 25 & 30 & 4.6 \\
0.001 & 5 & 500 & 8400 & 5.4 \\
0.0005 & 4 & 1200 & 54000 & 5.1 \\
\hline
\end{tabular}

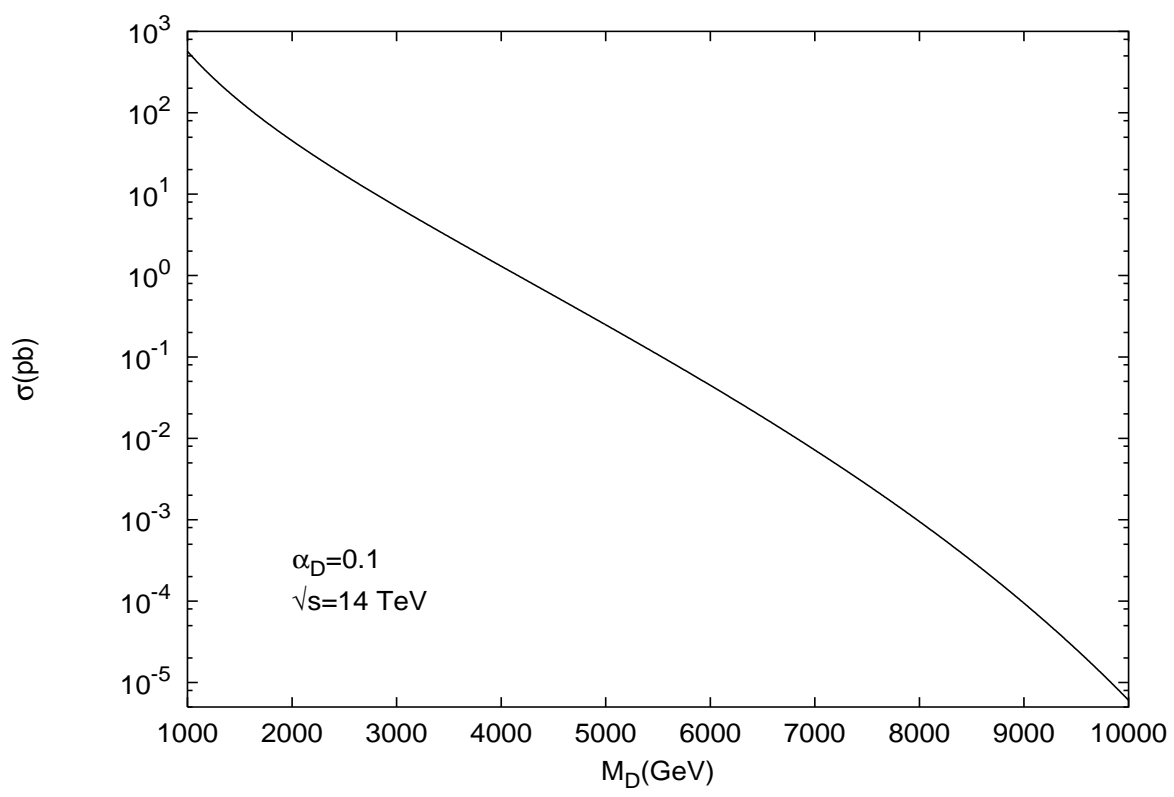

Figure 1: Total cross section versus diquark mass for $\alpha_{D}=0.1$. 


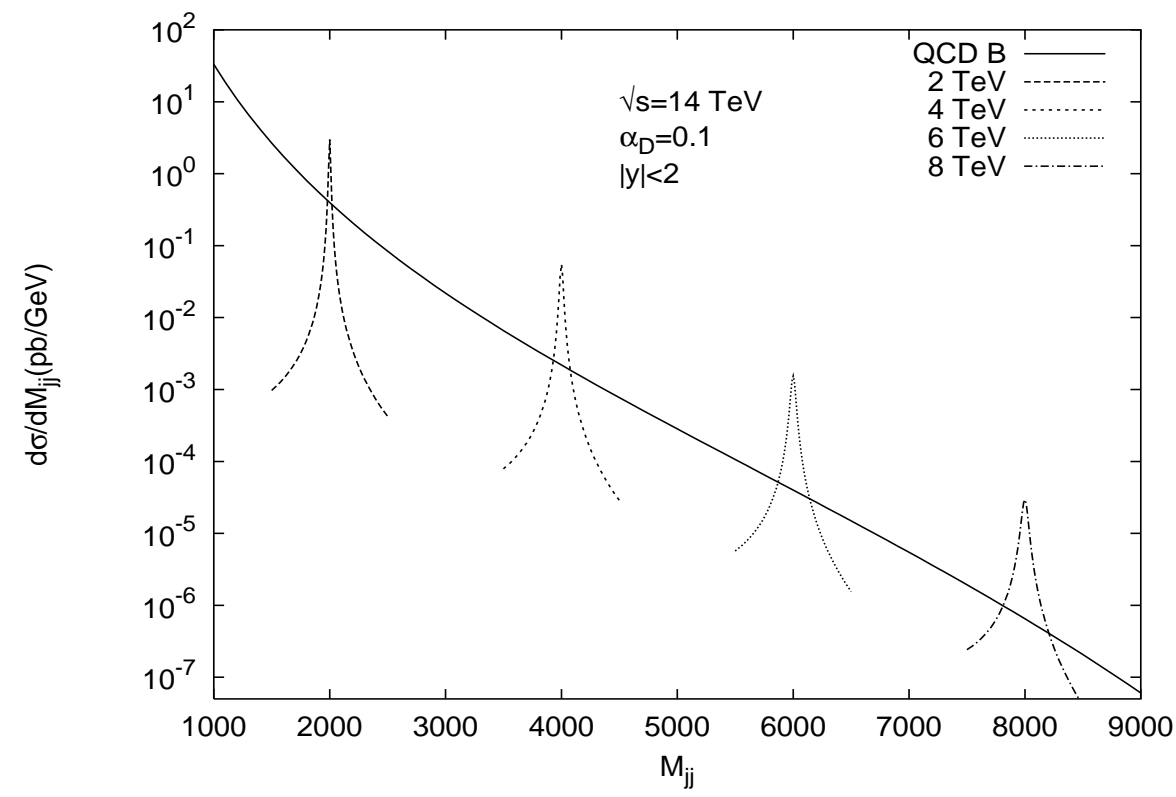

Figure 2: Dijet invariant mass distribution for $M_{D}=2,4,6,8 \mathrm{TeV}$ superimposed over the QCD background.

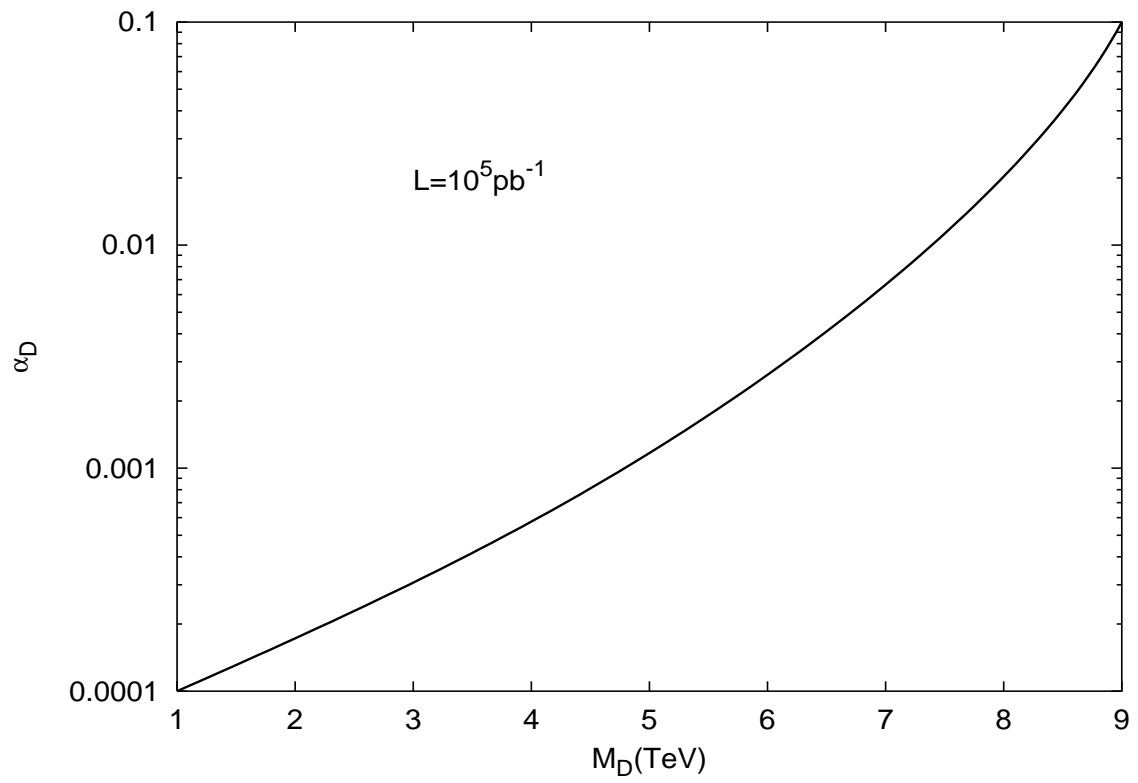

Figure 3: Attainable limits for vector diquark $D_{2}$ in $\alpha_{D^{-}} M_{D}$ plane. 


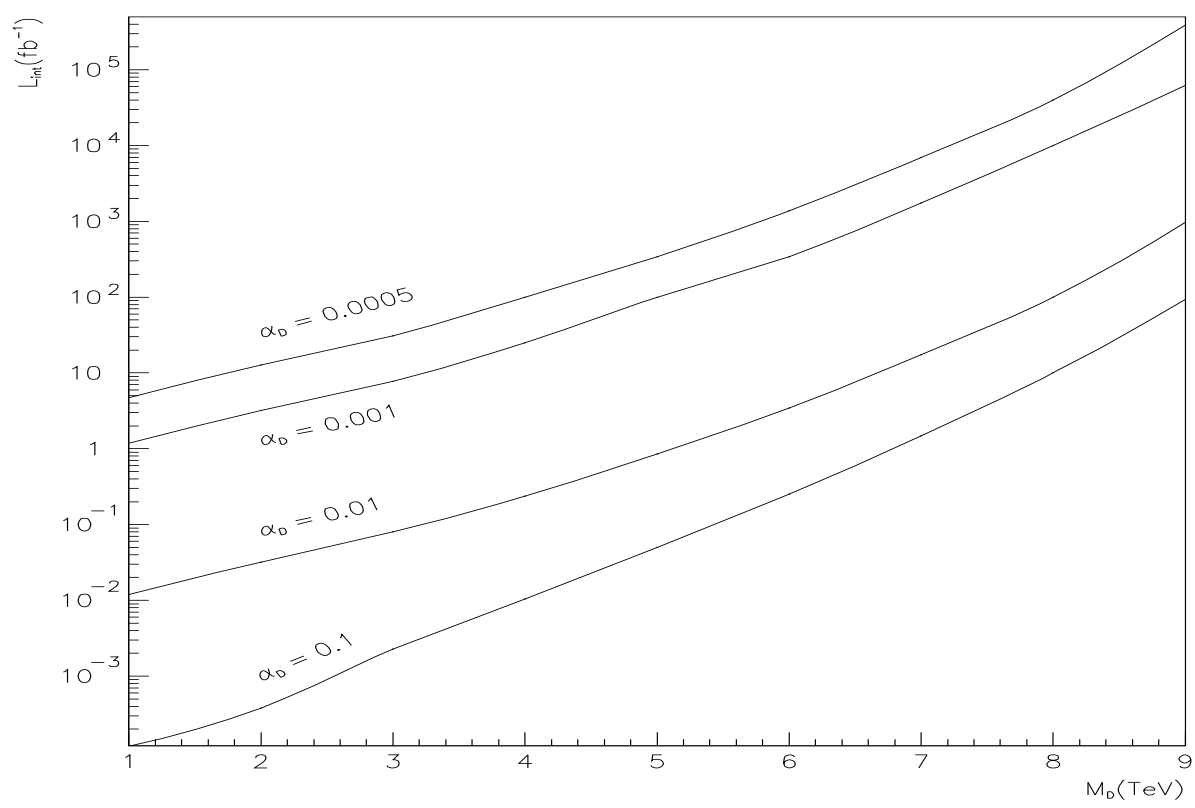

Figure 4: The minimum integrated luminosities, needed to satisfy the adopted discovery criteria, as a function of $M_{D}$ for various $\alpha_{D}$ values. 